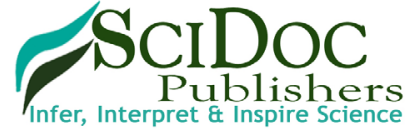

International Journal of Food Science, Nutrition and Dietetics (IJFS)

ISSN 2326-3350

\title{
Effects of Regimen Diet, without or with Lemon and Cumin Supplementation, On Lipid Profile and Endothelium Function in Obese and Overweight Patients
}

\author{
Research Article
}

Samah, A.S. Abdel-Shafy ${ }^{1 *}$, Seham A.M. Tharwat ${ }^{2}$, Ashraf. A. Abdel-Megeid ${ }^{2}$, Randa Salam ${ }^{3}$, Rehab H. Gab Allah ${ }^{4}$, Mary, W. Fawzy ${ }^{3}$

${ }^{1}$ Graduate Student, Nutrition and Food Science Dept. Faculty of Home Economics, Helwan University Egypt.

${ }^{2}$ Department of Nutrition and Food Science, Faculty of Home Economics, Helwan University Egypt.

${ }^{3}$ Department of Internal Medicine, Cairo University, Egypt.

${ }^{4}$ Dairy Technology Department, Food Technology Research Institute,Agricultural Research Center (A.R.C),Giza,Egypt.

\section{Abstract}

The aim of this study was to investigate the effects of regimen diet (RD) without or with containing yogurt supplemented with lemon, Cumin and Combination between them $(1: 1 \mathrm{w} / \mathrm{w})$ on lipid profile, Arterial atherosclerosis and endothelial dysfunction, to evaluate premature atheroscl-erosis in those patients Arandom sample of 100 patients (50 male and 50 female) with obesity of overweight selected from Kasr El Aini Hospital out patients, with age from 30-45 years, randomly patients were divided into 5 groups, each group includes 20 patients, 10 male and 10 female. Body weight and blood samples were collected from all participants at baseline and after 12 weeks to determine MBI, Lipid profile total cholesterol, (TC), Triglycerides (TG) LDL-c, VLDL-c and HDL-c, Carotid artery duplex, and Brachial artery flow media dilation. The first group Kept on their usual diet and considered as a control (+ve) group.

The second group treated only with regimen diet (RD) depend on BMI (1200 K.Cal for obese or 1500 K.Cal for overweight patients). The third group treated with the same (RD) Plus a dose of Cumin in amount of $5 \mathrm{~g} / \mathrm{d}$ in 12 weeks. The fourth group on (RD) plus a dose of lemon $5 \mathrm{~g} / \mathrm{d}$. The fifth group received (RD) plus a mixed dose $(1: 1 \mathrm{w} / \mathrm{w})$ of $2.5 \mathrm{~g} / \mathrm{d}$ Cumin powder plus $2.5 \mathrm{~g} / \mathrm{d}$ lemon. All supplementation with cumin and lemon were incorporated to skim milk yogurt product into their (RD) for 6 days/ week, 20 minutes before breakfast and lunch for 12 weeks. After 12 weeks of regimen dietary treatments. Results revealed that regimen diet containing skim milk yogurt containing cumin, lemon and mixed cumin plus lemon $(1: 1 \mathrm{w} / \mathrm{w})$ induced a high significant decrease in total cholesterol, triglycerides LDL-c, and VLDL-c. While results revealed a significant increase in HDL-c level, as compared to at baseline and as compared to (+ve) which treated with only (RD).

There was statistically difference between all groups, and the control ( $+v e)$ in flow mediated dilation of the brachial artery between all groups and $(+\mathrm{ve})$ control group without $(\mathrm{RD})$ our results indicate that $(+\mathrm{ve})$ group treated with $(\mathrm{RD})$ containing yogurt supplemented with mixed cumin plus lemon $(1: 1) \mathrm{w} / \mathrm{w}$ recorded the better values. There was statistically improvement in intima media thickness as compared to (+ve) control group without (RD). In conclusion our results indicated that (RD) containing skim milk yogurt supplemented with mixed of cumin plus lemon $(1: 1) \mathrm{w} / \mathrm{w}$ induced a high significant decrease in TC, LDL-c VLDL-c and triglycerides while increase HDL-c. Combination of Cumin plus lemon $(1: 1 \mathrm{w} / \mathrm{w})$ raise the protective effect of either component alone in preventing premature atherosclerosis in obese patients.

\section{Introduction}

The Prevalence of obesity is globally increasing and associated with a higher mortality [3]. Obesity is a major risk factor of Cardiovascular disease [14]. Many studies have proved that obesity is a major public health problem that result in decreased life expectancy in younger age groups. BMI itself, even without other anthropometric measures (e.g., waist circumference, waist-to- hip ratio), is a strong predictor for overall mortality. BMI above 22.5 $-25 \mathrm{~kg} / \mathrm{m}^{2}$ results in progressive increase in mortality which mainly related to cardiovascular disease [4].

The vascular endothelium plays a crucial role in the control of vascular homeostasis, providing a physical barrier between Lumen

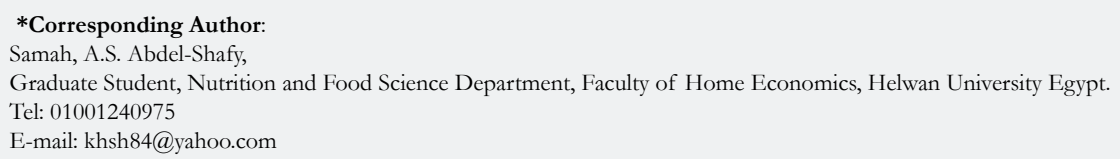

Citation: Samah, A.S. Abdel-Shafy, Seham A.M. Tharwat, Ashraf. A. Abdel-Megeid, Randa Salam, Rehab H. Gab Allah, Mary, W. Fawzy. Effects of Regimen Diet, without or with Lemon and Cumin Supplementation, On Lipid Profile and Endothelium Function in Obese and Overweight Patients. Int J Food Sci Nutr Diet. 2020;9(4):462-469. doi: http://dx.doi.org/10.19070/2326-3350-2000082

Copyright: Samah, A.S. Abdel-Shafy ${ }^{\circ}$ 2020. This is an open-access article distributed under the terms of the Creative Commons Attribution License, which permits unrestricted use, distribution and reproduction in any medium, provided the original author and source are credited. 
and the vessel wall, the endothelium actively regulates the basal vascular tone and reactivity in physiological conditions. Endothelial dysfunction, Causes and altered ability of the endothelium to maintain vascular homeostasis through the release of endothelium derived relaxing factors and endothelium derived contracting factors, is present in obesity and associated comorbidities [15].

Brachial flow mediation dilatation is the most widely measure of endothelial function Even that brachial FMD is variable, it has been associated with both CV risk factors and CV events [18].

\section{Subjects and Methods}

A random sample of 100 patients (50 male and 50 female with overweight or obesity were selected from Kasr El-Aini Hospital out patients with age from 30-45 years, with a body mass index of $>25:<30 \mathrm{Kg} / \mathrm{M}^{2}$ and of $30 \mathrm{Kg} / \mathrm{M}^{2}$ for overweight and obese men and women respectively, lack of any history disease and special drug consumption. Table (1) Shows the characteristics of experimental subjects:

Criteria for subject selection No previous treatment regimens in the past three months and lack of weight change by more than $2 \mathrm{Kg}$ in the last months were included.

\section{Exclusion criteria are as Follows}

Having allergic reactions to cumin and failure to the prescribed diet.

\section{Experimental Design}

The patients were randomly divided into 5 groups, each group consists of 20 subjects with overweight or obesity 10 male and 10 female, means age $30-45 \%$ Regimen dietary treatment as the following:

Group (1): Kept on their usual diet and considered as a control positive group (+ve).

Group (2): Received only regimen diet (RD) depend on BMI (< $30 \mathrm{MBI}$ ) consider lean and were fed on a (RD) contain (1500 K$\mathrm{Cal} / \mathrm{d})$, and $>30 \mathrm{MBI}$ consider obese and were on regimen diet contain $1200 \mathrm{~K} . \mathrm{Cal} / \mathrm{d}$.

Group (3): Received the same (RD) as group 2 plus a dose of Cumin in amount of $5 \mathrm{~g} / \mathrm{d}$ in 12 week.

Group (4): Received the same (RD) plus a dose of $5 \mathrm{~g}$ lemon/d in 12 weeks.

Group (5): Received the same (RD) plus a dose of $2.5 \mathrm{~g} / \mathrm{d}$ Cumin mixed with a dose of $2.5 \mathrm{~g} / \mathrm{d}$ Lemon $(1: 1 \mathrm{w} / \mathrm{w})$ all Cumin and lemon supplementation incorporated into skim milk yogurt product into their regimen diet for 6 days/ week, 20 minutes before breakfast and lunch for 12 weeks.

The selected physical parameters such as dietary pattern and family history of obesity were assessed before and after intervention. Baseline data were collected with the help of interview schedule and physical parameters were assessed by using standard measurement scales.

Anthropometric Assessment: Weight, height and body mass in- dex calculated according to Mitch and Klahy, (1993), as the weight $(\mathrm{Kg}) /$ height $\left(\mathrm{M}^{2}\right)$. The Prevalence of overweight and obesity was determined.

Dietary studies: 24 hours recall for 7 days before and after the intervention, food pattern and diet history were used.

The energy and nutrient content of the 24 hour were computed through the food composition table of National Institute (Food Composition tables for Egypt, 1996).

Biochemical analysis of Serum: Blood samples were collected from patients after $12 \mathrm{~h}$ overnight fast at baseline and after 12 weeks of the start to determine the following parameters:

Total cholesterol [12], Triglycerides [16], high density lipoprotein cholesterol HDL-c [10]. While serum low-density lipoprotein cholesterol (LDL-c) and very low-density lipoprotein cholesterol (VLDL-c) were calculated according to equation of [8].

Imaging Study: 1- Carotid artery duplex were done to all assess the carotid intima media thickness $2 \mathrm{~cm}$ just before the common carotid artery bifurcation by using the linear probe $7.5 \mathrm{MHZ}$ using HD 5000 machine, while the patient is lying flat, also we screen the internal carotid arteries searching for any carotid plaques.

2- Brachial artery flow media dilation: by a linear probe, 7.5 MHZ using a machine, the diameter of the brachial artery was measured while the patient is lying flat in bed and then it was re-measured after in response to an increase in blood flow during reactive hyperemia (induced by cuff inflation and then deflation). The cuff inflation period of 5 minutes is decided to produce adequate hyperemia to allow flow mediated dilatation, but not to compromise patient comfort. The usual scanning period used is 30 seconds before and 90 second after the cuff deflation. Then the percentage of dilation was calculated by equation:

All these imaging procedures was done at baseline and after regimen dietary treatments.

\section{Results and Discussion}

In another previous study [1], we clearly indicated that regimen diet containing yogurt supplemented with mixed of cumin plus lemon $(1: 1 \mathrm{w} / \mathrm{w})$ as a sources of alkaloid anthrquinon, coumarin, flavonoid glycoside in cumin and lemon polyphenols compounds significantly raise the effect of either component alone and induced a high significant decrease in BMI, Fasting blood glucose and glycated hemoglobine in obese and overweight patients. The results of this study clearly indicate the effect of (RD) containg yogurt supplemented with cumin or lemon and mixed between them on lipid profile and arterial atherosclerosis and endothelial dysfunction in obese and over weight patients:

\section{Effect of Different Regiment Diet Treatments on Lipid Pro- file}

Table ( $2 \& 3)$ shows the effect of different dietary treatments on (TC) in overweight and obese patients.

Statistical analysis of results revealed that $(+v e)$ male and female 
Table 1. Characteristics of Experimental Subject.

\begin{tabular}{|c|c|c|c|c|c|c|c|c|c|c|c|c|c|c|c|c|c|c|c|c|}
\hline $\begin{array}{l}\text { Groups } \\
\text { Treatment }\end{array}$ & \multicolumn{4}{|c|}{$\begin{array}{c}\text { Group -1- } \\
\text { \# Control A (usual diet) }\end{array}$} & \multicolumn{4}{|c|}{$\begin{array}{c}\text { Group -2- } \\
\text { \# Regimen diet }\end{array}$} & \multicolumn{4}{|c|}{$\begin{array}{c}\text { Group -3- } \\
\text { \# Regimen diet containing } \\
\text { cumin }\end{array}$} & \multicolumn{4}{|c|}{$\begin{array}{c}\text { Group -4- } \\
\text { \# Regimen diet containing } \\
\text { lemon }\end{array}$} & \multicolumn{4}{|c|}{$\begin{array}{c}\text { Group -5- } \\
\text { \# Regimen diet containing } \\
\text { cumin and lemon }\end{array}$} \\
\hline \multirow[t]{2}{*}{ Parameters } & \multicolumn{2}{|c|}{$\begin{array}{c}\text { Over- } \\
\text { weight }\end{array}$} & \multicolumn{2}{|c|}{ Obese } & \multicolumn{2}{|c|}{$\begin{array}{c}\text { Over- } \\
\text { Weight }\end{array}$} & \multicolumn{2}{|c|}{ Obese } & \multicolumn{2}{|c|}{$\begin{array}{c}\text { Over- } \\
\text { weight }\end{array}$} & \multicolumn{2}{|c|}{ Obese } & \multicolumn{2}{|c|}{$\begin{array}{c}\text { Over- } \\
\text { weight }\end{array}$} & \multicolumn{2}{|c|}{ Obese } & \multicolumn{2}{|c|}{$\begin{array}{c}\text { Over- } \\
\text { weight }\end{array}$} & \multicolumn{2}{|c|}{ Obese } \\
\hline & $\mathbf{M}$ & $F$ & & F & M & $F$ & & F & $\mathbf{M}$ & F & $\mathbf{M}$ & $\mathrm{F}$ & $\mathbf{M}$ & $F$ & $\mathbf{M}$ & $F$ & $\mathbf{M}$ & $F$ & $\mathbf{M}$ & $F$ \\
\hline $\begin{array}{c}\text { BMI at } \\
\text { baseline } \\
\text { (mean } \pm \text { SD) }\end{array}$ & $\begin{array}{c}27 \\
\pm 1.6\end{array}$ & $\begin{array}{r}27.5 \\
\pm 1.3\end{array}$ & $\begin{array}{l}32.7 \\
\pm 1.6\end{array}$ & $\begin{array}{c}33.1 \\
\pm 1.3\end{array}$ & $\begin{array}{l}27.7 \\
\pm 1.3\end{array}$ & $\begin{array}{l}27.8 \\
\pm 1.3\end{array}$ & $\begin{array}{l}32.4 \\
\pm 1.6\end{array}$ & $\begin{array}{l}33.7 \\
\pm 1.0\end{array}$ & $\begin{array}{l}27.5 \\
\pm 1.4\end{array}$ & $\begin{array}{l}28.1 \\
\pm 1.0\end{array}$ & $\begin{array}{l}33.8 \\
\pm 0.6\end{array}$ & $\begin{array}{c}33 \\
\pm 2.0\end{array}$ & $\begin{array}{l}27.2 \\
\pm 1.5\end{array}$ & $\begin{array}{l}27.8 \\
\pm 1.5\end{array}$ & $\begin{array}{r}33.3 \\
\pm 1.7\end{array}$ & $\begin{array}{l}33.1 \\
\pm 1.4\end{array}$ & $\begin{array}{r}28.3 \\
\pm 1.2\end{array}$ & $\begin{array}{c}27 \\
\pm 0.9\end{array}$ & $\begin{array}{l}31.8 \\
\pm 3.6\end{array}$ & $\begin{array}{r}32.4 \\
\pm 1.4\end{array}$ \\
\hline $\begin{array}{c}\text { Age }(\mathrm{Y}) \\
(\text { mean } \pm \mathrm{SD})\end{array}$ & $\begin{array}{r}38.8 \\
\pm 3.4\end{array}$ & $\begin{array}{r}40.2 \\
\pm 3.6\end{array}$ & $\begin{array}{c}39.4 \\
\pm 2.4\end{array}$ & $\begin{array}{l}41.6 \\
=2.9\end{array}$ & $\begin{array}{r}39.2 \\
\pm 3.7\end{array}$ & $\begin{array}{l}40.4 \\
\pm 3.1\end{array}$ & $\begin{array}{r}39.6 \\
\pm 3.3 \\
\end{array}$ & $\begin{array}{r}39 \\
\pm 1.4\end{array}$ & $\begin{array}{r}37.6 \\
\pm 2.7\end{array}$ & $\begin{array}{r}38 \\
\pm 2.2\end{array}$ & $\begin{array}{r}42 \\
\pm 1.4\end{array}$ & $\begin{array}{r}39.6 \\
+3.8 \\
\end{array}$ & $\begin{array}{r}37.6 \\
\pm 2.7\end{array}$ & $\begin{array}{r}41.2 \\
\pm 2.8\end{array}$ & $\begin{array}{r}43.6 \\
\pm 1.7\end{array}$ & $\begin{array}{r}38.6 \\
\pm 3.1\end{array}$ & $\begin{array}{r}37.4 \\
\pm 2.9\end{array}$ & $\begin{array}{l}41.4 \\
\pm 2.4\end{array}$ & $\begin{array}{r}39.4 \\
\pm 3.5\end{array}$ & $\begin{array}{r}39 \\
+2.2 \\
\end{array}$ \\
\hline
\end{tabular}

Table 2. Effects of Regimen diet with or without supplemented yogurt with cumin or lemon and Mixed Between them (1:1w/w) on Lipid Fractions (Total cholesterol) in Male overweight and obese patients at basely and after 12 weeks.

\begin{tabular}{|c|c|c|c|c|c|c|c|c|c|c|c|c|c|c|}
\hline \multirow{2}{*}{$\begin{array}{c}\begin{array}{c}\text { Groups Treat- } \\
\text { ment }\end{array} \\
\begin{array}{c}\text { Parameters } \\
(\mathrm{mg} / \mathrm{dl})\end{array}\end{array}$} & \multicolumn{2}{|c|}{$\begin{array}{l}\text { Group -1- } \\
\text { \# Control A } \\
\text { (usual diet) }\end{array}$} & \multicolumn{2}{|c|}{$\begin{array}{c}\text { Group -2- } \\
\text { \# Regimen diet }\end{array}$} & \multicolumn{2}{|c|}{$\begin{array}{c}\text { Group -3- } \\
\text { \# Regimen } \\
\text { diet containing } \\
\text { cumin }\end{array}$} & \multicolumn{2}{|c|}{$\begin{array}{c}\text { Group -4- } \\
\text { \# Regimen } \\
\text { diet containing } \\
\text { lemon }\end{array}$} & \multicolumn{2}{|c|}{$\begin{array}{c}\text { Group -5- } \\
\text { \# Regimen diet } \\
\text { containing cumin } \\
\text { and lemon }\end{array}$} & \multirow[t]{2}{*}{$\begin{array}{l}\text { Anova } \\
\text { Over } \\
\text {-weight }\end{array}$} & \multirow[t]{2}{*}{$\begin{array}{c}\text { LSD } \\
\text { Over- } \\
\text { weight }\end{array}$} & \multirow[t]{2}{*}{$\begin{array}{l}\text { Anova } \\
\text { obese }\end{array}$} & \multirow[t]{2}{*}{$\begin{array}{l}\text { LSD } \\
\text { obese }\end{array}$} \\
\hline & $\begin{array}{c}\text { Over- } \\
\text { weight }\end{array}$ & Obese & $\begin{array}{c}\text { Over- } \\
\text { weight }\end{array}$ & Obese & $\begin{array}{c}\text { Over- } \\
\text { weight }\end{array}$ & Obese & $\begin{array}{c}\text { Over- } \\
\text { weight }\end{array}$ & Obese & $\begin{array}{c}\text { Over- } \\
\text { weight }\end{array}$ & Obese & & & & \\
\hline $\begin{array}{l}\text { Total cholesterol } \\
\text { at baseline (mean } \\
\pm \mathrm{SD})\end{array}$ & $\begin{array}{c}233 \\
\pm 51.3\end{array}$ & $\begin{array}{c}246.4 \\
\pm 40.7\end{array}$ & $\begin{array}{c}237 \\
\pm 36.1\end{array}$ & $\begin{array}{l}240.8 \\
\pm 35.5\end{array}$ & $\begin{array}{l}266.6 \\
\pm 17.2\end{array}$ & $\begin{array}{l}256.2 \\
\pm 13.2\end{array}$ & $\begin{array}{l}279.8 \\
\pm 24.8\end{array}$ & $\begin{array}{c}250 \\
\pm 35.6\end{array}$ & $\begin{array}{l}251.6 \\
\pm 27.3\end{array}$ & $\begin{array}{l}251.8 \\
\pm 58.2\end{array}$ & 0.18 & - & 0.978 & - \\
\hline $\begin{array}{l}\text { Total cholesterol } \\
\text { after } 12 \text { Weeks } \\
(\text { mean } \pm \text { SD) }\end{array}$ & $\begin{array}{c}\mathrm{a} \\
279.8 \\
279.8 \\
\pm 45.7\end{array}$ & $\begin{array}{c}a \\
276.6 \\
276.6 \\
\pm 52.9\end{array}$ & $\begin{array}{c}\mathrm{b} \\
216.4 \\
216.4 \\
\pm 35.2\end{array}$ & $\begin{array}{c}\mathrm{b} \\
222 \\
222 \\
\pm 29.9\end{array}$ & $\begin{array}{c}\mathrm{b} \\
186.6 \\
186.6 \\
\pm 16.7\end{array}$ & $\begin{array}{c}\mathrm{bc} \\
190.6 \\
179.6 \\
\pm 12.0\end{array}$ & $\begin{array}{c}\mathrm{b} \\
184.8 \\
180.6 \\
\pm 8.3\end{array}$ & $\begin{array}{c}c \\
179.6 \\
177.8 \\
\pm 8.5\end{array}$ & $\begin{array}{c}\mathrm{b} \\
180.6 \\
184.8 \\
\pm 8.7\end{array}$ & $\begin{array}{c}c \\
177.8 \\
190.6 \\
\pm 12.3\end{array}$ & $0.000^{* *}$ & 36.11 & $0.000^{* *}$ & 37.587 \\
\hline T. test & $0.000^{* *}$ & 0.216 & $0.020^{*}$ & $0.013^{*}$ & $0.002^{* *}$ & $0.001^{* *}$ & $0.001 * *$ & $0.010^{* *}$ & $0.002^{* *}$ & 0.071 & & & & \\
\hline
\end{tabular}

Mean with the same letter are not significantly different

(*) significant at $\leq 0.05$

(**) highly significant at $\leq 0.01$

Table 3. Effects of Regimen diet with or without supplemented yogurt with cumin or lemon and Mixed Between them (1:1w/w) on Lipid Fractions (Total cholesterol ) in Female overweight and obese patients at baseline and after 12 weeks.

\begin{tabular}{|c|c|c|c|c|c|c|c|c|c|c|c|c|c|c|}
\hline \multirow{2}{*}{$\begin{array}{c}\text { Groups } \\
\text { Treatment }\end{array}$} & \multicolumn{2}{|c|}{$\begin{array}{c}\text { Group -1- } \\
\text { \# Control A } \\
\text { (usual diet) }\end{array}$} & \multicolumn{2}{|c|}{$\begin{array}{c}\text { Group -2- } \\
\text { \# Regimen diet }\end{array}$} & \multicolumn{2}{|c|}{$\begin{array}{c}\text { Group -3- } \\
\text { \# Regimen diet } \\
\text { containing cumin }\end{array}$} & \multicolumn{2}{|c|}{$\begin{array}{c}\text { Group -4- } \\
\text { \# Regimen } \\
\text { diet containing } \\
\text { lemon }\end{array}$} & \multicolumn{2}{|c|}{$\begin{array}{c}\text { Group -5- } \\
\text { \# Regimen diet } \\
\text { containing cumin } \\
\text { and lemon }\end{array}$} & \multirow{2}{*}{$\begin{array}{c}\text { Anova } \\
\text { over- } \\
\text { weight }\end{array}$} & \multirow{2}{*}{$\begin{array}{c}\text { LSD } \\
\text { Over- } \\
\text { weight }\end{array}$} & \multirow[t]{2}{*}{$\begin{array}{l}\text { Anova } \\
\text { obese }\end{array}$} & \multirow[t]{2}{*}{$\begin{array}{l}\text { LSD } \\
\text { obese }\end{array}$} \\
\hline & $\begin{array}{c}\text { Over- } \\
\text { weight }\end{array}$ & Obese & $\begin{array}{c}\text { Over- } \\
\text { weight }\end{array}$ & Obese & $\begin{array}{c}\text { Over- } \\
\text { weight }\end{array}$ & Obese & $\begin{array}{c}\text { Over- } \\
\text { weight }\end{array}$ & Obese & $\begin{array}{c}\text { Over- } \\
\text { weight }\end{array}$ & Obese & & & & \\
\hline $\begin{array}{l}\text { Total choles- } \\
\text { terol } \\
\text { at baseline } \\
\text { (mean } \pm \mathrm{SD})\end{array}$ & $\begin{array}{c}245 \\
\pm 28.0\end{array}$ & $\begin{array}{c}251 \\
\pm 15.8\end{array}$ & $\begin{array}{c}230 \\
\pm 34.8\end{array}$ & $\begin{array}{l}255.6 \\
\pm 52.4\end{array}$ & $\begin{array}{l}275.8 \\
\pm 27.0\end{array}$ & $\begin{array}{c}255 \\
\pm 28.8\end{array}$ & $\begin{array}{c}264 \\
\pm 28.4\end{array}$ & $\begin{array}{l}249.4 \\
\pm 9.2\end{array}$ & $\begin{array}{l}262.4 \\
\pm 40.9\end{array}$ & $\begin{array}{l}282.2 \\
\pm 21.2\end{array}$ & 0.226 & - & 0.414 & - \\
\hline $\begin{array}{l}\text { Total choles- } \\
\text { terol after } 12 \\
\text { Weeks } \\
\text { (mean } \pm \text { SD) }\end{array}$ & $\begin{array}{c}a \\
255 \\
255 \\
\pm 15.3\end{array}$ & $\begin{array}{c}a \\
268.8 \\
268.8 \\
\pm 30.3\end{array}$ & $\begin{array}{c}\mathrm{b} \\
216.4 \\
216.4 \\
\pm 33.2\end{array}$ & $\begin{array}{c}\mathrm{b} \\
224.8 \\
224.8 \\
\pm 36.1\end{array}$ & $\begin{array}{c}c \\
189.6 \\
189.6 \\
\pm 10.9\end{array}$ & $\begin{array}{c}c \\
194.4 \\
194.4 \\
\pm 4.3\end{array}$ & $\begin{array}{c}c \\
182 \\
181.2 \\
\pm 7.5\end{array}$ & $\begin{array}{c}c \\
190.8 \\
190.6 \\
\pm 7.7\end{array}$ & $\begin{array}{c}c \\
181.2 \\
182 \\
\pm 9.6\end{array}$ & $\begin{array}{c}c \\
190.6 \\
190.8 \\
\pm 8.5\end{array}$ & $0.000^{* *}$ & 23.58 & $0.000^{* *}$ & 28.73 \\
\hline T. test & 0.503 & 0.184 & $0.026^{*}$ & $0.023^{*}$ & $0.002^{* *}$ & $0.007^{* *}$ & $0.002^{* *}$ & $0.000^{* *}$ & $0.007^{* *}$ & $0.000 * *$ & & & & \\
\hline
\end{tabular}

Mean with the same letter are not significantly different

$(*)$ significant at $\leq 0.05$

(**) highly significant at $\leq 0.01$

obese and overweight groups kept on their usual diet (3200 \pm 10 $\mathrm{K} . \mathrm{Cal} / \mathrm{d})$ recorded a significant increase $(\mathrm{P}<0.01)$ in $(\mathrm{TC})$ values as compared to at base line. On the other hand results showed that (+ve) group of male and female obese and overweight treated only with regimen diet $(1200$ or $1500 \mathrm{~K} . \mathrm{Cal} / \mathrm{d})$ induced a significant decrease $(\mathrm{P}<0.05)$ in $(\mathrm{TC})$ as compared to at base line.

Statistical analysis of results revealed that (+ve) male and female obese and overweight groups kept on their usual diet (3200 \pm 10 $\mathrm{K} . \mathrm{Cal} / \mathrm{d}$ ) recorded a significant increase $(\mathrm{P}<0.01)$ in $(\mathrm{TC})$ values as compared to at base line. On the other hand results showed that (+ve) group male and female obese and overweight treated only with regimen diet (1200 or $1500 \mathrm{~K} . \mathrm{Cal} / \mathrm{d})$ induced a signifi- cant decrease $(\mathrm{P}<0.05)$ in $(\mathrm{TC})$ as compared to at baseline.

On the other hand our results revealed that all obese and overweight groups treated with (RD) containing yogurt supplemented with Cumin or lemon and mixed between them $(1: 1 \mathrm{w} / \mathrm{w})$ induced a high significant decrease $(\mathrm{P}<0.01)$ in TC level.

In this respect $(19 ; 1)$ demonstrated that hypocholesterolemic effect of cumin could be partly attributed to its glycoside saponins which prevent cholesterol absorption and increase its fecal excretion by interfering with its enterohepatic circulation. Moreover, Cumin seeds have a substantial amount of some phytosterols such as beta-sistosterol, delta 5-avenasterol, and delta 7-avenas- 
terol. Cumin has also stigmasterol, campesterol and lanosterol in small amount. Phytosterols displace cholesterol from intestinal micelles reducing absorbable cholesterol.

Concerning the effect of lemon in reducing (TC) Yaghmaie et al., (2011) demonstrated that citrus peel essential oil in 50 and 100 $\mathrm{ml} / \mathrm{kg}$ doses demonstrated a significant reduction in TG, TC and LDL $(\mathrm{P}<0.01)$.

Effect of Regimen Diet with or without Yogurt Supplemented with Cumin Lemon and Mixed Between them (1:1w/w) on Serum Triglycerides in Overweight and Obese Patients

Table (4\&5) shows the effect of (RD) with Yogurt supplemented with lemon, cumin or without and mixed between them. Results revealed that male \& female obese $(+v e)$ control without dietary treatment, kept on their usual diet recorded a high significant increase $(\mathrm{P}<0.01)$ in triglycerides (TG) level while male obese group which treated only on $(\mathrm{RD})$ recorded a significant decrease $(\mathrm{P}<0.01)$ as compared with at baseline, while results revealed a significant decrease $(\mathrm{P}<0.05)$ recorded by male and female over weight groups which treated with regimen diet containing yogurt supplemented with cumin, While male obese groups on (RD) containing Yogurt supplemented with lemon induced high significant decrease $(\mathrm{P}<0.01)$ in serum $(\mathrm{TG})$ level. On the other side overweight male and obese \& overweight female groups which treated with $(\mathrm{RD})$ containing mixed Cumin plus lemon recorded a significant decrease $(\mathrm{P}<0.05)$ in $(\mathrm{TG})$ as compared to at baseline.

Effect of Regimen Diet with or Without Containing Yogurt Supplemented with Cumin Lemon and Mixed Between them on Serum Lipoprotein Cholesterol Fractions in Overweight and Obese Patients

Table (6 \& 7) shows the effect different (RD) with or without yogurt supplemented with cumin, lemon and mixed between them on HDL-c level.

Result shows that control (+ve) group kept on their usual diet recorded significant decrease $(\mathrm{P}<0.01)$ in HDL-c values as compared to at baseline. On the other hand (+ve) of over weight male group which treated with only with $(\mathrm{RD})$ recorded a significant increase $(\mathrm{P}<0.01)$ in HDL-c as compared to $(+\mathrm{ve})$ control and as compared to at baseline.

On the other side groups of over weight and obese female \& male which treated with (RD) containing yogurt supplemented with cumin induced a significant increase $(\mathrm{P}<0.05)$ in HDL-c level our results revealed that male and female obese and overweight groups treated with $(\mathrm{RD})$ containing supplemented yogurt with

Table 4. Effects of Regimen diet with or without supplemented yogurt with cumin or lemon and Mixed Between them (1:1w/w) on Triglycerides level in Male overweight and obese patients at baseline and after 12 weeks.

\begin{tabular}{|c|c|c|c|c|c|c|c|c|c|c|c|c|c|c|}
\hline \multirow{2}{*}{$\begin{array}{c}\text { Groups } \\
\text { Treatment } \\
\begin{array}{c}\text { Parameters } \\
(\mathrm{mg} / \mathrm{dl})\end{array}\end{array}$} & \multicolumn{2}{|c|}{$\begin{array}{l}\text { Group -1- } \\
\text { \# Control A } \\
\text { (usual diet) }\end{array}$} & \multicolumn{2}{|c|}{$\begin{array}{c}\text { Group -2- } \\
\text { \# Regimen diet }\end{array}$} & \multicolumn{2}{|c|}{$\begin{array}{c}\text { Group -3- } \\
\text { \# Regimen } \\
\text { diet containing } \\
\text { cumin }\end{array}$} & \multicolumn{2}{|c|}{$\begin{array}{c}\text { Group -4- } \\
\text { \# Regimen diet } \\
\text { containing lemon }\end{array}$} & \multicolumn{2}{|c|}{$\begin{array}{c}\text { Group -5- } \\
\text { \# Regimen diet } \\
\text { containing cumin } \\
\text { and lemon }\end{array}$} & \multirow{2}{*}{$\begin{array}{c}\text { Anova } \\
\text { over- } \\
\text { weight }\end{array}$} & \multirow{2}{*}{$\begin{array}{c}\text { LSD } \\
\text { over- } \\
\text { weight }\end{array}$} & \multirow[t]{2}{*}{$\begin{array}{l}\text { Anova } \\
\text { obese }\end{array}$} & \multirow[t]{2}{*}{$\begin{array}{l}\text { LSD } \\
\text { obese }\end{array}$} \\
\hline & $\begin{array}{c}\text { Over- } \\
\text { weight }\end{array}$ & Obese & $\begin{array}{c}\text { Over- } \\
\text { weight }\end{array}$ & Obese & $\begin{array}{c}\text { Over- } \\
\text { weight }\end{array}$ & Obese & $\begin{array}{c}\text { Over- } \\
\text { weight }\end{array}$ & Obese & $\begin{array}{c}\text { Over- } \\
\text { weight }\end{array}$ & Obese & & & & \\
\hline $\begin{array}{l}\text { Triglycerides } \\
\text { at baseline } \\
\text { (mean } \pm \mathrm{SD})\end{array}$ & $\begin{array}{c}100.6 \\
\pm 13.2\end{array}$ & $\begin{array}{l}110.4 \\
\pm 8.6\end{array}$ & $\begin{array}{c}100.4 \\
\pm 13.0\end{array}$ & $\begin{array}{c}111.6 \\
\pm 11.5\end{array}$ & $\begin{array}{c}111 \\
\pm 6.0\end{array}$ & $\begin{array}{c}112 \\
\pm 7.0\end{array}$ & $\begin{array}{l}114.6 \\
\pm 8.6\end{array}$ & $\begin{array}{l}119.8 \\
\pm 6.1\end{array}$ & $\begin{array}{c}109 \\
\pm 5.3\end{array}$ & $\begin{array}{l}111.2 \\
\pm 8.2\end{array}$ & 0.116 & - & 0.414 & \\
\hline $\begin{array}{l}\text { Triglycerides } \\
\text { after } 12 \text { Weeks } \\
(\text { mean } \pm \mathrm{SD})\end{array}$ & $\begin{array}{c}\mathrm{a} \\
128.8 \\
128.8 \\
\pm 12.3\end{array}$ & $\begin{array}{c}a \\
171.2 \\
171.2 \\
\pm 17.2\end{array}$ & $\begin{array}{c}\mathrm{b} \\
102.2 \\
98 \\
\pm 3.5\end{array}$ & $\begin{array}{c}\mathrm{b} \\
102.2 \\
99.8 \\
\pm 8.7\end{array}$ & $\begin{array}{c}\mathrm{b} \\
98 \\
93 \\
\pm 10.5\end{array}$ & $\begin{array}{c}\mathrm{b} \\
99.8 \\
93.2 \\
\pm 14.2\end{array}$ & $\begin{array}{c}\mathrm{b} \\
93 \\
102.2 \\
\pm 19.3\end{array}$ & $\begin{array}{c}\mathrm{b} \\
93.2 \\
102.2 \\
\pm 11.1\end{array}$ & $\begin{array}{c}\mathrm{b} \\
92.2 \\
92.2 \\
\pm 9.9\end{array}$ & $\begin{array}{c}\mathrm{b} \\
92.2 \\
91 \\
\pm 13.6\end{array}$ & $0.001 * *$ & 16.1 & $0.000^{* *}$ & 17.52 \\
\hline T. test & 0.056 & $0.002^{* *}$ & 0.716 & $0.002^{* *}$ & $0.019 *$ & 0.057 & 0.261 & $0.017^{*}$ & $0.018^{*}$ & $0.007 * *$ & & & & \\
\hline
\end{tabular}

Mean with the same letter are not significantly different

$(*)$ significant at $\leq 0.05$

(**) highly significant at $\leq 0.01$

Table 5. Effects of Regimen diet with or without supplemented yogurt with cumin or le and Mixed Between them (1:1w/w) mon on Triglycerides level in Female overweight and obese patients at baseline and after 12 weeks.

\begin{tabular}{|c|c|c|c|c|c|c|c|c|c|c|c|c|c|c|}
\hline \multirow{2}{*}{$\begin{array}{c}\text { Groups } \\
\text { Treatment }\end{array}$} & \multicolumn{2}{|c|}{$\begin{array}{l}\text { Group -1- } \\
\text { \# Control A } \\
\text { (usual diet) }\end{array}$} & \multicolumn{2}{|c|}{$\begin{array}{c}\text { Group -2- } \\
\text { \# Regimen diet }\end{array}$} & \multicolumn{2}{|c|}{$\begin{array}{c}\text { Group -3- } \\
\text { \# Regimen } \\
\text { diet containing } \\
\text { cumin }\end{array}$} & \multicolumn{2}{|c|}{$\begin{array}{c}\text { Group -4- } \\
\text { \# Regimen } \\
\text { diet containing } \\
\text { lemon }\end{array}$} & \multicolumn{2}{|c|}{$\begin{array}{c}\text { Group -5- } \\
\text { \# Regimen diet } \\
\text { containing cumin } \\
\text { and lemon }\end{array}$} & \multirow{2}{*}{$\begin{array}{l}\text { Anova } \\
\text { over- } \\
\text { weight }\end{array}$} & \multirow{2}{*}{$\begin{array}{c}\text { LSD } \\
\text { over- } \\
\text { weight }\end{array}$} & \multirow[t]{2}{*}{$\begin{array}{l}\text { Anova } \\
\text { obese }\end{array}$} & \multirow[t]{2}{*}{$\begin{array}{l}\text { LSD } \\
\text { obese }\end{array}$} \\
\hline & $\begin{array}{l}\text { Over- } \\
\text { weight }\end{array}$ & Obese & $\begin{array}{l}\text { Over- } \\
\text { weight }\end{array}$ & Obese & $\begin{array}{c}\text { Over- } \\
\text { weight }\end{array}$ & Obese & $\begin{array}{c}\text { Over- } \\
\text { weight }\end{array}$ & Obese & $\begin{array}{c}\text { Over- } \\
\text { weight }\end{array}$ & Obese & & & & \\
\hline $\begin{array}{l}\text { Triglycerides } \\
\text { at baseline } \\
(\text { mean } \pm \text { SD) }\end{array}$ & $\begin{array}{r}111.4 \\
\pm 5.5\end{array}$ & $\begin{array}{r}98 \\
\pm 15.6\end{array}$ & $\begin{array}{c}93.4 \\
\pm 14.8\end{array}$ & $\begin{array}{c}98 \\
\pm 18.6\end{array}$ & $\begin{array}{l}113.4 \\
\pm 3.9\end{array}$ & $\begin{array}{c}114 \\
\pm 11.3\end{array}$ & $\begin{array}{l}123.4 \\
\pm 6.7\end{array}$ & $\begin{array}{l}112.8 \\
\pm 2.2\end{array}$ & $\begin{array}{l}108.4 \\
\pm 6.9\end{array}$ & $\begin{array}{l}114.6 \\
\pm 3.2\end{array}$ & $0.000 * *$ & - & 0.068 & - \\
\hline $\begin{array}{l}\text { Triglycerides } \\
\text { after } 12 \text { Weeks } \\
(\text { mean } \pm \text { SD) }\end{array}$ & $\begin{array}{c}a \\
147.8 \\
147.8 \\
\pm 18.4\end{array}$ & $\begin{array}{c}a \\
147.6 \\
147.6 \\
\pm 11.4\end{array}$ & $\begin{array}{c}\mathrm{b} \\
100 \\
90.8 \\
\pm 12.4\end{array}$ & $\begin{array}{c}\mathrm{b} \\
100.4 \\
91 \\
\pm 12.2\end{array}$ & $\begin{array}{c}\mathrm{b} \\
98.4 \\
98.4 \\
\pm 7.6\end{array}$ & $\begin{array}{c}\mathrm{b} \\
96.4 \\
100.4 \\
\pm 20.3\end{array}$ & $\begin{array}{c}\mathrm{b} \\
97.2 \\
100 \\
\pm 14.7\end{array}$ & $\begin{array}{c}\mathrm{b} \\
96.4 \\
96.4 \\
\pm 11.5\end{array}$ & $\begin{array}{c}\mathrm{b} \\
90.8 \\
97.2 \\
\pm 6.6\end{array}$ & $\begin{array}{c}\mathrm{b} \\
91 \\
94.4 \\
+10.0\end{array}$ & $0.000^{* *}$ & 16.78 & $0.000^{* *}$ & 17.88 \\
\hline T. test & $0.013^{*}$ & $0.000^{* *}$ & 0.152 & 0.192 & $0.017^{*}$ & 0.079 & 0.069 & $0.020^{*}$ & $0.015^{*}$ & $0.017^{*}$ & & & & \\
\hline
\end{tabular}

Mean with the same letter are not significantly different

$(*)$ significant at $\leq 0.05$

(**) highly significant at $\leq 0.01$ 
Table 6. Effects of Regimen diet with or without supplemented yogurt with cumin or lemon and Mixed Between them (1:1w/w) on LipoProtein Cholesterol (HDL) Fractions in Male overweight and obese patients at baseline and after 12 weeks.

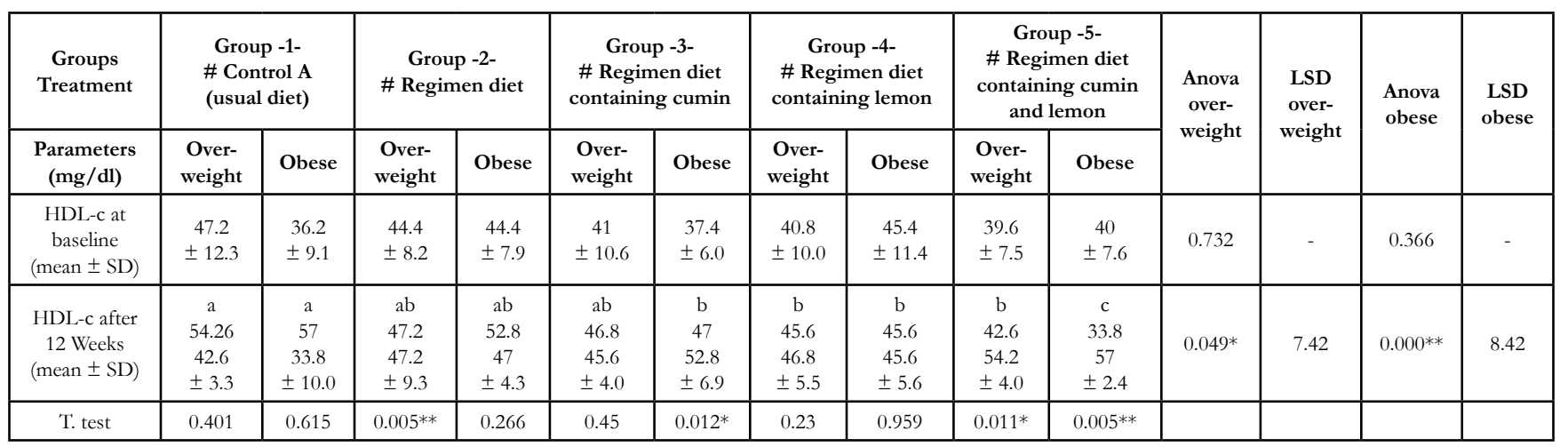

Mean with the same letter are not significantly different

$(*)$ significant at $\leq 0.05$

${ }^{(* *)}$ highly significant at $\leq 0.01$

Table 7. Effects of Regimen diet with or without supplemented yogurt with cumin or lemon and Mixed Between them (1:1w/w) on LipoProtein Cholesterol(HDL) Fractions in Female overweight and obese patients at baseline and after 12 weeks.

\begin{tabular}{|c|c|c|c|c|c|c|c|c|c|c|c|c|c|c|}
\hline \multirow{2}{*}{$\begin{array}{c}\text { Groups } \\
\text { Treatment }\end{array}$} & \multicolumn{2}{|c|}{$\begin{array}{l}\text { Group -1- } \\
\text { \# Control A } \\
\text { (usual diet) }\end{array}$} & \multicolumn{2}{|c|}{$\begin{array}{c}\text { Group -2- } \\
\text { \# Regimen diet }\end{array}$} & \multicolumn{2}{|c|}{$\begin{array}{c}\text { Group -3- } \\
\text { \# Regimen } \\
\text { diet containing } \\
\text { cumin }\end{array}$} & \multicolumn{2}{|c|}{$\begin{array}{c}\text { Group -4- } \\
\text { \# Regimen } \\
\text { diet containing } \\
\text { lemon }\end{array}$} & \multicolumn{2}{|c|}{$\begin{array}{c}\text { Group -5- } \\
\text { \# Regimen } \\
\text { diet containing } \\
\text { cumin and lemon }\end{array}$} & \multirow{2}{*}{$\begin{array}{l}\text { Anova } \\
\text { over- } \\
\text { weight }\end{array}$} & \multirow{2}{*}{$\begin{array}{c}\text { LSD } \\
\text { over- } \\
\text { weight }\end{array}$} & \multirow[t]{2}{*}{$\begin{array}{l}\text { Anova } \\
\text { obese }\end{array}$} & \multirow[t]{2}{*}{$\begin{array}{l}\text { LSD } \\
\text { obese }\end{array}$} \\
\hline & $\begin{array}{l}\text { Over- } \\
\text { weight }\end{array}$ & Obese & $\begin{array}{c}\text { Over- } \\
\text { weight }\end{array}$ & Obese & $\begin{array}{c}\text { Over- } \\
\text { weight }\end{array}$ & Obese & $\begin{array}{c}\text { Over- } \\
\text { weight }\end{array}$ & Obese & $\begin{array}{l}\text { Over- } \\
\text { weight }\end{array}$ & Obese & & & & \\
\hline $\begin{array}{c}\text { HDL-c at } \\
\text { baseline } \\
(\text { mean } \pm \text { SD) }\end{array}$ & $\begin{array}{c}38.2 \\
\pm 6.2\end{array}$ & $\begin{array}{c}32.2 \\
\pm 3.7\end{array}$ & $\begin{array}{c}46.4 \\
\pm 10.6\end{array}$ & $\begin{array}{c}40.6 \\
\pm 12.6\end{array}$ & $\begin{array}{r}36.2 \\
\pm 4.4\end{array}$ & $\begin{array}{l}40.2 \\
\pm 7.3\end{array}$ & $\begin{array}{c}44.4 \\
\pm 13.3\end{array}$ & $\begin{array}{l}49.8 \\
\pm 8.4\end{array}$ & $\begin{array}{l}42.8 \\
\pm 5.7\end{array}$ & $\begin{array}{c}41 \\
\pm 9.3\end{array}$ & 0.353 & - & 0.072 & - \\
\hline $\begin{array}{l}\text { HDL-c after } \\
12 \text { Weeks } \\
(\text { mean } \pm S D)\end{array}$ & $\begin{array}{c}a \\
53 \\
28.4 \\
\pm 4.6\end{array}$ & $\begin{array}{c}a \\
55.6 \\
31.2 \\
\pm 5.1\end{array}$ & $\begin{array}{c}\mathrm{a} \\
52.2 \\
52.2 \\
\pm 10.6\end{array}$ & $\begin{array}{c}\mathrm{ab} \\
53.6 \\
46 \\
+10.9\end{array}$ & $\begin{array}{c}\mathrm{a} \\
48.4 \\
48.2 \\
\pm 2.5\end{array}$ & $\begin{array}{c}\mathrm{ab} \\
50.2 \\
50.2 \\
\pm 5.2\end{array}$ & $\begin{array}{c}\mathrm{a} \\
48.2 \\
48.4 \\
\pm 5.4\end{array}$ & $\begin{array}{c}\mathrm{b} \\
36 \\
53.6 \\
\pm 4.3\end{array}$ & $\begin{array}{c}\mathrm{b} \\
28.4 \\
53 \\
\pm 2.5\end{array}$ & $\begin{array}{c}c \\
31.2 \\
55.6 \\
\pm 3.0\end{array}$ & $0.000^{* *}$ & 7.78 & $0.000^{* *}$ & 8.33 \\
\hline T. test & 0.1 & 0.546 & 0.232 & 0.072 & $0.015^{*}$ & $0.049 *$ & 0.515 & 0.29 & $0.004 * *$ & $0.043^{*}$ & & & & \\
\hline
\end{tabular}

Mean with the same letter are not significantly differen

$(*)$ significant at $\leq 0.05$

$\left.{ }^{* *}\right)$ highly significant at $\leq 0.01$

mixed (cumin and lemon 1:1 w/w) induced a high significant increase in HDL-c values.

Concerning low-density lipoprotein (LDL-c) and very low density lipoprotein cholesterol (VLDL-c), Table (8\&9) (10\&11) shows the effects of different dietary treatments on LDL-c \& VLDL-c levels, with different dietary treatments.

Results revealed that control (+ve) male and female groups kept on their usual diet recorded a significant increase $(\mathrm{P}<0.05)$ in LDL-c and VLDL-c as compared to at baseline.

While male and female overweight and obese groups treated with only $(\mathrm{RD})$ recorded a significant decrease $(\mathrm{P}<0.01)$ in LDL-c and VLDL-c. On the other hand all groups which treated with (RD) containing cumin, lemon and mixed between them induced a significant decrease as compared to at baseline.

In this respect [19], reported that cumin powder reduced serum level of fasting cholesterol, triglycerides and LDL-c and increased HDL-c.

Concerning the effect of feeding lemon Miyake, (1997) suggested that lemon polyphenols is beneficial for the suppression of diet- induced obesity, and improvement of insulin resistance and lipid metabolism. Polyphenol; which may mean that stimulation of fatty acid oxidation, rather than the suppression of lipogenesis, is the predominant contribution that lemon polyphenol make [17]. demonstrated that treated with citrus peel essential oil induce a significant reduction in triglycerides, cholesterol LDL.

Effect of Regimen Diet with or without Yogurt Supplemented with Cumin, Lemon and Mixed Between them On Arterial Atherosclerosis and Enothelial Dysfunction in Obese Patients

Tables (12\&13) shows values of flow mediated dilation (FD\%).

Result revealed that all our obese patients at the baseline of the study were hypercholesteremic with elevates LDL-c and reduced HDL-c, with increased carotid intima media thickness (Table 12 \& 13)

In our study we found that at baseline all groups had elevated lipid profile and increased carotid intima media thickness confirming that obesity perse is a major risk factor for atherosclerosis.

There was statistical difference in flow mediated dilation of the 
Table 8. Effects of Regimen diet with or without supplemented yogurt with cumin or lemon and Mixed Between them (1:1w/w) on Serum LipoProteins (LDL-C) in Male overweight and obese patients at baseline and after 12 weeks.

\begin{tabular}{|c|c|c|c|c|c|c|c|c|c|c|c|c|c|c|}
\hline \multirow{2}{*}{$\begin{array}{c}\begin{array}{c}\text { Groups } \\
\text { Treatment }\end{array} \\
\begin{array}{c}\text { Parameters } \\
(\mathrm{mg} / \mathrm{dl})\end{array}\end{array}$} & \multicolumn{2}{|c|}{$\begin{array}{l}\text { Group -1- } \\
\text { \# Control A } \\
\text { (usual diet) }\end{array}$} & \multicolumn{2}{|c|}{$\begin{array}{c}\text { Group -2- } \\
\text { \# Regimen diet }\end{array}$} & \multicolumn{2}{|c|}{$\begin{array}{c}\text { Group -3- } \\
\text { \# Regimen diet } \\
\text { containing cumin }\end{array}$} & \multicolumn{2}{|c|}{$\begin{array}{c}\text { Group -4- } \\
\text { \# Regimen diet } \\
\text { containing lemon }\end{array}$} & \multicolumn{2}{|c|}{$\begin{array}{c}\text { Group -5- } \\
\text { \# Regimen diet } \\
\text { containing cumin } \\
\text { and lemon }\end{array}$} & \multirow[t]{2}{*}{$\begin{array}{l}\text { Anova } \\
\text { Over- } \\
\text { weight }\end{array}$} & \multirow[t]{2}{*}{$\begin{array}{c}\text { LSD } \\
\text { Over- } \\
\text { weigh }\end{array}$} & \multirow[t]{2}{*}{$\begin{array}{l}\text { Anova } \\
\text { obese }\end{array}$} & \multirow[t]{2}{*}{$\begin{array}{l}\text { LSD } \\
\text { obese }\end{array}$} \\
\hline & $\begin{array}{c}\text { Over- } \\
\text { weight }\end{array}$ & Obese & $\begin{array}{c}\text { Over- } \\
\text { weight }\end{array}$ & Obese & $\begin{array}{c}\text { Over- } \\
\text { weight }\end{array}$ & Obese & $\begin{array}{c}\text { Over- } \\
\text { weight }\end{array}$ & Obese & $\begin{array}{c}\text { Over- } \\
\text { weight }\end{array}$ & Obese & & & & \\
\hline $\begin{array}{c}\text { LDL-c at } \\
\text { baseline } \\
\text { (mean } \pm \mathrm{SD})\end{array}$ & $\begin{array}{r}163.6 \\
\pm 60.3\end{array}$ & $\begin{array}{r}207.5 \\
\pm 37.1\end{array}$ & $\begin{array}{l}186.2 \\
\pm 35.0\end{array}$ & $\begin{array}{r}203.2 \\
\pm 33.1\end{array}$ & $\begin{array}{l}142.4 \\
\pm 26.6\end{array}$ & $\begin{array}{c}188.2 \\
\pm 16.3\end{array}$ & $\begin{array}{r}172.5 \\
\pm 46.1\end{array}$ & $\begin{array}{c}141 \\
\pm 35.0\end{array}$ & $\begin{array}{r}194.8 \\
\pm 32.5\end{array}$ & $\begin{array}{l}200.2 \\
\pm 58.7\end{array}$ & 0.344 & - & 0.075 & - \\
\hline $\begin{array}{l}\text { LDL-c after } \\
12 \text { Weeks } \\
(\text { mean } \pm \text { SD) }\end{array}$ & $\begin{array}{c}a \\
174.18 \\
174.2 \\
\pm 64.7\end{array}$ & $\begin{array}{c}\mathrm{a} \\
197.8 \\
197.9 \\
\pm 41.8\end{array}$ & $\begin{array}{c}\mathrm{ab} \\
165.6 \\
165.6 \\
\pm 37.4\end{array}$ & $\begin{array}{c}\mathrm{a} \\
185.54 \\
185.5 \\
\pm 22.9\end{array}$ & $\begin{array}{c}\mathrm{bc} \\
126.66 \\
107.9 \\
\pm 6.3\end{array}$ & $\begin{array}{c}\mathrm{b} \\
124.52 \\
118.7 \\
\pm 3.2\end{array}$ & $\begin{array}{c}\mathrm{bc} \\
120.4 \\
120.4 \\
\pm 7.4\end{array}$ & $\begin{array}{c}b \\
119.96 \\
120 \\
\pm 7.6\end{array}$ & $\begin{array}{c}\mathrm{bc} \\
107.94 \\
126.7 \\
\pm 16.1\end{array}$ & $\begin{array}{c}b \\
118.66 \\
124.5 \\
\pm 12.1\end{array}$ & $0.023^{*}$ & 45.44 & $0.000 * *$ & 29.41 \\
\hline T. test & 0.636 & 0.662 & $0.010^{* *}$ & 0.053 & $0.026^{*}$ & $0.001^{* *}$ & $0.050^{*}$ & 0.23 & $0.013^{*}$ & 0.061 & & & & \\
\hline
\end{tabular}

Mean with the same letter are not significantly different

(*) significant at $\leq 0.05$

(**) highly significant at $\leq 0.01$

Table 9. Effects of Regimen diet with or without supplemented yogurt with cumin or lemo and Mixed Between them (1:1w/w) on Serum Lipo Proteins (LDL-C) in Female overweight and obese patients at baseline and after 12 weeks.

\begin{tabular}{|c|c|c|c|c|c|c|c|c|c|c|c|c|c|c|}
\hline \multirow{2}{*}{$\begin{array}{c}\begin{array}{c}\text { Groups } \\
\text { Treatment }\end{array} \\
\begin{array}{c}\text { Parameters } \\
(\mathrm{mg} / \mathrm{dl})\end{array}\end{array}$} & \multicolumn{2}{|c|}{$\begin{array}{c}\text { Group -1- } \\
\text { \# Control A (usual } \\
\text { diet) }\end{array}$} & \multicolumn{2}{|c|}{$\begin{array}{c}\text { Group -2- } \\
\text { \# Regimen diet }\end{array}$} & \multicolumn{2}{|c|}{$\begin{array}{c}\text { Group -3- } \\
\text { \# Regimen diet } \\
\text { containing cumin }\end{array}$} & \multicolumn{2}{|c|}{$\begin{array}{c}\text { Group -4- } \\
\text { \# Regimen } \\
\text { diet containing } \\
\text { lemon }\end{array}$} & \multicolumn{2}{|c|}{$\begin{array}{c}\text { Group -5- } \\
\text { \# Regimen diet } \\
\text { containing cumin } \\
\text { and lemon }\end{array}$} & \multirow[t]{2}{*}{$\begin{array}{c}\text { Anova } \\
\text { Over- } \\
\text { weight }\end{array}$} & \multirow[t]{2}{*}{$\begin{array}{c}\text { LSD } \\
\text { Over- } \\
\text { weighr }\end{array}$} & \multirow[t]{2}{*}{$\begin{array}{l}\text { Anova } \\
\text { obese }\end{array}$} & \multirow[t]{2}{*}{$\begin{array}{l}\text { LSD } \\
\text { obese }\end{array}$} \\
\hline & $\begin{array}{l}\text { Over- } \\
\text { weight }\end{array}$ & Obese & $\begin{array}{l}\text { Over- } \\
\text { Weight }\end{array}$ & Obese & $\begin{array}{l}\text { Over- } \\
\text { Weight }\end{array}$ & Obese & $\begin{array}{l}\text { Over- } \\
\text { Weight }\end{array}$ & Obese & $\begin{array}{c}\text { Over } \\
\text { Weight }\end{array}$ & Obese & & & & \\
\hline $\begin{array}{c}\text { LDL-c at } \\
\text { baseline } \\
\text { (mean } \pm \mathrm{SD})\end{array}$ & $\begin{array}{r}184.6 \\
\pm 28.5\end{array}$ & $\begin{array}{r}199.2 \\
\pm 14.4\end{array}$ & $\begin{array}{r}160.9 \\
\pm 41.2\end{array}$ & $\begin{array}{r}191.4 \\
\pm 63.4\end{array}$ & $\begin{array}{r}213.2 \\
\pm 26.3\end{array}$ & $\begin{array}{c}170.1 \\
\pm 35.8\end{array}$ & $\begin{array}{r}175.5 \\
\pm 56.4\end{array}$ & $\begin{array}{l}145.4 \\
\pm 41.6\end{array}$ & $\begin{array}{c}199.2 \\
\pm 16.2\end{array}$ & $\begin{array}{l}231.5 \\
\pm 17.2\end{array}$ & 0.226 & - & $0.027 *$ & - \\
\hline $\begin{array}{l}\text { LDL-c after } \\
12 \text { Weeks } \\
(\text { mean } \pm \text { SD) }\end{array}$ & $\begin{array}{c}a \\
205.3 \\
205.3 \\
\pm 30.9\end{array}$ & $\begin{array}{c}\mathrm{a} \\
220.14 \\
220.1 \\
\pm 22.7\end{array}$ & $\begin{array}{c}b \\
150.9 \\
150.9 \\
\pm 38.4\end{array}$ & $\begin{array}{c}B \\
160.34 \\
160.3 \\
\pm 49.9\end{array}$ & $\begin{array}{c}\mathrm{bc} \\
123.8 \\
123.8 \\
\pm 3.9\end{array}$ & $\begin{array}{c}c \\
126.16 \\
126.2 \\
\pm 6.1\end{array}$ & $\begin{array}{c}c \\
119.8 \\
119.2 \\
\pm 7.7\end{array}$ & $\begin{array}{c}c \\
118 \\
117.1 \\
\pm 4.2\end{array}$ & $\begin{array}{c}c \\
119.2 \\
119.8 \\
\pm 4.5\end{array}$ & $\begin{array}{c}c \\
117.1 \\
118 \\
\pm 12.4\end{array}$ & $0.000^{* *}$ & 29.61 & $0.000 * *$ & 33.44 \\
\hline T. test & $0.041 *$ & 0.059 & $0.010^{* *}$ & 0.014* & $0.003^{* *}$ & $0.049 *$ & 0.08 & 0.2 & $0.000^{* *}$ & $0.001 * *$ & & & & \\
\hline
\end{tabular}

Mean with the same letter are not significantly different

$(*)$ significant at $\leq 0.05$

(**) highly significant at $\leq 0.01$

Table 10. Effects of Regimen diet with or without supplemented yogurt with cumin or lemon and Mixed Between them (1:1w/w) on Serum LipoProteins (VLDL-C) in Male overweight and obese patients at baseline and after 12 weeks.

\begin{tabular}{|c|c|c|c|c|c|c|c|c|c|c|c|c|c|c|}
\hline \multirow{2}{*}{$\begin{array}{c}\text { Groups } \\
\text { Treatment } \\
\begin{array}{c}\text { Parameters } \\
\text { (mg/dl) }\end{array} \\
\end{array}$} & \multicolumn{2}{|c|}{$\begin{array}{l}\text { Group -1- } \\
\text { \# Control A } \\
\text { (usual diet) }\end{array}$} & \multicolumn{2}{|c|}{$\begin{array}{c}\text { Group -2- } \\
\text { \# Regimen diet }\end{array}$} & \multicolumn{2}{|c|}{$\begin{array}{c}\text { Group -3- } \\
\text { \# Regimen } \\
\text { diet containing } \\
\text { cumin }\end{array}$} & \multicolumn{2}{|c|}{$\begin{array}{c}\text { Group -4- } \\
\text { \# Regimen } \\
\text { diet containing } \\
\text { lemon }\end{array}$} & \multicolumn{2}{|c|}{$\begin{array}{c}\text { Group -5- } \\
\text { \# Regimen } \\
\text { diet containing } \\
\text { cumin and lemon }\end{array}$} & \multirow[t]{2}{*}{$\begin{array}{c}\text { Anova } \\
\text { Over- } \\
\text { weight }\end{array}$} & \multirow[t]{2}{*}{$\begin{array}{l}\text { LSD } \\
\text { Over- } \\
\text { weight }\end{array}$} & \multirow[t]{2}{*}{$\begin{array}{l}\text { Anova } \\
\text { obese }\end{array}$} & \multirow[t]{2}{*}{$\begin{array}{l}\text { LSD } \\
\text { obese }\end{array}$} \\
\hline & $\begin{array}{c}\text { Over- } \\
\text { weight }\end{array}$ & Obese & $\begin{array}{c}\text { Over- } \\
\text { weight }\end{array}$ & Obese & $\begin{array}{l}\text { Over- } \\
\text { weight }\end{array}$ & Obese & $\begin{array}{c}\text { Over- } \\
\text { weight }\end{array}$ & Obese & $\begin{array}{c}\text { Over } \\
\text { weight }\end{array}$ & Obese & & & & \\
\hline $\begin{array}{l}\text { VLDL-c at } \\
\text { baseline } \\
\text { (mean } \pm \text { SD) }\end{array}$ & $\begin{array}{l}20.2 \\
\pm 2.7\end{array}$ & $\begin{array}{l}20.8 \\
\pm 2.2\end{array}$ & $\begin{array}{c}21.3 \\
\pm 1.3\end{array}$ & $\begin{array}{r}22.3 \\
\pm 2.5\end{array}$ & $\begin{array}{c}21 \\
\pm 2.4\end{array}$ & $\begin{array}{l}20.8 \\
\pm 1.4\end{array}$ & $\begin{array}{l}22.9 \\
\pm 1.7\end{array}$ & $\begin{array}{l}24.5 \\
\pm 3.3\end{array}$ & $\begin{array}{c}21 \\
\pm 0.8\end{array}$ & $\begin{array}{l}21.6 \\
\pm 0.9\end{array}$ & 0.303 & - & 0.086 & \\
\hline $\begin{array}{l}\text { VLDL-c after } \\
12 \text { Weeks } \\
(\text { mean } \pm \text { SD) }\end{array}$ & $\begin{array}{c}\mathrm{a} \\
23.4 \\
23.4 \\
\pm 2.1\end{array}$ & $\begin{array}{c}a \\
24.52 \\
24.5 \\
\pm 2.6\end{array}$ & $\begin{array}{c}\mathrm{b} \\
20 \\
20 \\
\pm 1.2\end{array}$ & $\begin{array}{c}\mathrm{b} \\
20.66 \\
20.7 \\
\pm 2.6\end{array}$ & $\begin{array}{c}\mathrm{b} \\
19.4 \\
18.7 \\
\pm 2.5\end{array}$ & $\begin{array}{c}\mathrm{b} \\
20.44 \\
19.3 \\
\pm 1.7\end{array}$ & $\begin{array}{c}\mathrm{b} \\
18.66 \\
19.4 \\
\pm 2.6\end{array}$ & $\begin{array}{c}\mathrm{b} \\
19.34 \\
20.4 \\
\pm 2.2\end{array}$ & $\begin{array}{c}\mathrm{b} \\
18.3 \\
18.3 \\
\pm 1.8\end{array}$ & $\begin{array}{c}\mathrm{b} \\
17.8 \\
17.8 \\
\pm 2.1\end{array}$ & $0.009 * *$ & 2.8 & $0.002^{* *}$ & 2.97 \\
\hline T. test & 0.104 & $0.006^{* *}$ & 0.091 & $0.033^{*}$ & $0.026^{*}$ & 0.162 & 0.055 & 0.061 & $0.035^{*}$ & $0.027^{*}$ & & & & \\
\hline
\end{tabular}

Mean with the same letter are not significantly different

$\left(^{*}\right)$ significant at $\leq 0.05$

(**) highly significant at $\leq 0.01$

brachial artery between all groups. Results revealed that the better values induced by groups which treated with (RD) containing yogurt supplemented with mixed cumin plus lemon, however there. Male and female obese and overweight groups treated with (RD) containing supplemented yogurt with lemon had better outcome as regards lipid profile, intima media thicknes, and brachial flow dilation parameters as compared to control (+ve) without (RD) and control (+ve) on (RD), and the same result was found in the group treated with $(\mathrm{RD})$ containing yogurt supplemented with cumin alone $(2 ; 5)$

In this respect Hooper et al., (2008) demonstrated that an inverse 
Table 11. Effects of Regimen diet with or without supplemented yogurt with cumin or lemon and Mixed Between them (1:1w/w) on Serum LipoProteins (VLDL-C) in Female overweight and obese patients at baseline and after 12 weeks.

\begin{tabular}{|c|c|c|c|c|c|c|c|c|c|c|c|c|c|c|}
\hline \multirow{2}{*}{$\begin{array}{c}\text { Groups } \\
\text { Treatment }\end{array}$} & \multicolumn{2}{|c|}{$\begin{array}{c}\text { Group -1- } \\
\text { \# Control A (usual } \\
\text { diet) }\end{array}$} & \multicolumn{2}{|c|}{$\begin{array}{c}\text { Group -2- } \\
\text { \# Regimen diet }\end{array}$} & \multicolumn{2}{|c|}{$\begin{array}{c}\text { Group -3- } \\
\text { \# Regimen } \\
\text { diet containing } \\
\text { cumin }\end{array}$} & \multicolumn{2}{|c|}{$\begin{array}{c}\text { Group -4- } \\
\text { \# Regimen diet } \\
\text { containing lemon }\end{array}$} & \multicolumn{2}{|c|}{$\begin{array}{c}\text { Group -5- } \\
\text { \# Regimen diet } \\
\text { containing cumin } \\
\text { and lemon }\end{array}$} & \multirow[t]{2}{*}{$\begin{array}{c}\text { Anova } \\
\text { over- } \\
\text { weight }\end{array}$} & \multirow[t]{2}{*}{$\begin{array}{c}\text { LSD } \\
\text { over- } \\
\text { weight }\end{array}$} & \multirow[t]{2}{*}{$\begin{array}{l}\text { Anova } \\
\text { obese }\end{array}$} & \multirow[t]{2}{*}{$\begin{array}{l}\text { LSD } \\
\text { obese }\end{array}$} \\
\hline & $\begin{array}{c}\text { Over- } \\
\text { weight }\end{array}$ & Obese & $\begin{array}{l}\text { Over- } \\
\text { weight }\end{array}$ & Obesel & $\begin{array}{c}\text { Over- } \\
\text { weight }\end{array}$ & Obese & $\begin{array}{c}\text { Over- } \\
\text { weight }\end{array}$ & Obese & $\begin{array}{c}\text { Over- } \\
\text { weight }\end{array}$ & Obese & & & & \\
\hline $\begin{array}{c}\text { VLDL-c } \\
\text { at baseline } \\
\text { (mean } \pm \mathrm{SD})\end{array}$ & $\begin{array}{r}22.2 \\
\pm 1.1\end{array}$ & $\begin{array}{r}19.6 \\
\pm 3.1\end{array}$ & $\begin{array}{c}18.7 \\
\pm 3.0\end{array}$ & $\begin{array}{l}20.8 \\
\pm 2.1\end{array}$ & $\begin{array}{r}22.6 \\
\pm 1.1\end{array}$ & $\begin{array}{r}22.5 \\
\pm 2.2\end{array}$ & $\begin{array}{l}23.9 \\
\pm 1.7\end{array}$ & $\begin{array}{l}22.6 \\
\pm 0.4\end{array}$ & $\begin{array}{r}22.4 \\
\pm 1.2\end{array}$ & $\begin{array}{l}22.7 \\
\pm 0.8\end{array}$ & $0.002^{* *}$ & - & 0.087 & - \\
\hline $\begin{array}{l}\text { VLDL-c after } \\
12 \text { Weeks } \\
\text { (mean } \pm \text { SD) }\end{array}$ & $\begin{array}{c}a \\
24.3 \\
24.3 \\
\pm 1.6 \\
\end{array}$ & $\begin{array}{c}a \\
25.06 \\
25.1 \\
\pm 1.7 \\
\end{array}$ & $\begin{array}{c}\mathrm{b} \\
17.76 \\
17.3 \\
\pm 2.5 \\
\end{array}$ & $\begin{array}{c}\mathrm{b} \\
19.5 \\
19.3 \\
\pm 1.9 \\
\end{array}$ & $\begin{array}{c}\mathrm{b} \\
19.48 \\
19.5 \\
\pm 2.0 \\
\end{array}$ & $\begin{array}{c}\mathrm{b} \\
19.34 \\
18.7 \\
\pm 1.9 \\
\end{array}$ & $\begin{array}{c}\mathrm{b} \\
18.96 \\
19 \\
\pm 3.4 \\
\end{array}$ & $\begin{array}{c}b \\
18.66 \\
19.5 \\
\pm 1.8 \\
\end{array}$ & $\begin{array}{c}\mathrm{b} \\
17.3 \\
19.8 \\
\pm 1.4 \\
\end{array}$ & $\begin{array}{c}\mathrm{b} \\
18.6 \\
18.6 \\
\pm 1.6 \\
\end{array}$ & $0.002^{* *}$ & 3.019 & $0.000^{* *}$ & 2.33 \\
\hline T. test & $0.012^{*}$ & $0.010^{* *}$ & $0.011 *$ & 0.001 ** & $0.038^{*}$ & $0.011 *$ & 0.082 & $0.013^{*}$ & $0.021 *$ & $0.006^{* *}$ & & & & \\
\hline
\end{tabular}

Mean with the same letter are not significantly different (*) significant at $\leq 0.05$

(**) highly significant at $\leq 0.01$

Table 12. Effects of Regimen diet with or without supplemented yogurt with cumin or lemon and Mixed Between them (1:1w/w)on Endothelium Function (Flow Dilation) in Male overweight and obese patients at baseline and after 12 weeks.

\begin{tabular}{|c|c|c|c|c|c|c|c|c|c|c|c|c|c|c|}
\hline \multirow{2}{*}{$\begin{array}{c}\text { Groups Treatment } \\
\text { Parameters }\end{array}$} & \multicolumn{2}{|c|}{$\begin{array}{c}\text { Group -1- } \\
\text { \# Control A } \\
\text { (usual diet) }\end{array}$} & \multicolumn{2}{|c|}{$\begin{array}{c}\text { Group -2- } \\
\text { \# Regimen diet }\end{array}$} & \multicolumn{2}{|c|}{$\begin{array}{c}\text { Group -3- } \\
\text { \# Regimen } \\
\text { diet containing } \\
\text { cumin }\end{array}$} & \multicolumn{2}{|c|}{$\begin{array}{c}\text { Group -4- } \\
\text { \# Regimen } \\
\text { diet containing } \\
\text { lemon }\end{array}$} & \multicolumn{2}{|c|}{$\begin{array}{c}\text { Group -5- } \\
\text { \# Regimen } \\
\text { diet contain- } \\
\text { ing cumin and } \\
\text { lemon }\end{array}$} & \multirow[t]{2}{*}{$\begin{array}{c}\text { Anova } \\
\text { over- } \\
\text { weight }\end{array}$} & \multirow[t]{2}{*}{$\begin{array}{c}\text { LSD } \\
\text { over- } \\
\text { weight }\end{array}$} & \multirow[t]{2}{*}{$\begin{array}{c}\text { Anova } \\
\text { obese }\end{array}$} & \multirow[t]{2}{*}{$\begin{array}{c}\text { LSD } \\
\text { obese }\end{array}$} \\
\hline & $\begin{array}{c}\text { Over- } \\
\text { weight }\end{array}$ & Obese & $\begin{array}{c}\text { Over- } \\
\text { weight }\end{array}$ & Obese & $\begin{array}{c}\text { Over- } \\
\text { weight }\end{array}$ & Obese & $\begin{array}{c}\text { Over- } \\
\text { weight }\end{array}$ & Obese & $\begin{array}{c}\text { Over- } \\
\text { weight }\end{array}$ & Obese & & & & \\
\hline $\begin{array}{c}\text { Endothelium Func- } \\
\text { tion (Flow Dilation) } \\
\text { at baseline } \\
\text { (mean } \pm \mathrm{SD} \text { ) }\end{array}$ & $\begin{array}{l}23.8 \\
\pm 9.7\end{array}$ & $\begin{array}{c}11.1 \\
\pm 3.9\end{array}$ & $\begin{array}{c}13.7 \\
\pm 18.3\end{array}$ & $\begin{array}{l}18.9 \\
\pm 15.1\end{array}$ & $\begin{array}{c}19.8 \\
\pm 11.8\end{array}$ & $\begin{array}{l}21.8 \\
\pm 9.6\end{array}$ & $\begin{array}{c}19.5 \\
\pm 24.4\end{array}$ & $\begin{aligned} & 23 \\
\pm & 15.6\end{aligned}$ & $\begin{array}{c}11.5 \\
\pm 13.0\end{array}$ & $\begin{aligned} & 18 \\
\pm & 12.3\end{aligned}$ & 0.777 & - & 0.683 & - \\
\hline $\begin{array}{l}\text { Endothelium Func- } \\
\text { tion (Flow Dilation) } \\
\text { after } \\
12 \text { Weeks } \\
\text { (mean } \pm \text { SD) }\end{array}$ & $\begin{array}{c}\mathrm{a} \\
37.71 \\
16.7 \\
\pm 0.8\end{array}$ & $\begin{array}{c}\text { A } \\
25.45 \\
9.2 \\
\pm 2.4\end{array}$ & $\begin{array}{c}\mathrm{ab} \\
22.73 \\
15.3 \\
\pm 18.0\end{array}$ & $\begin{array}{c}a \\
23.21 \\
19.7 \\
\pm 15.3\end{array}$ & $\begin{array}{c}\mathrm{ab} \\
18.36 \\
22.7 \\
\pm 13.1\end{array}$ & $\begin{array}{c}\mathrm{ab} \\
22.79 \\
25.5 \\
\pm 6.1\end{array}$ & $\begin{array}{c}\mathrm{b} \\
15.32 \\
18.4 \\
\pm 19.1\end{array}$ & $\begin{array}{c}\mathrm{ab} \\
19.64 \\
22.8 \\
\pm 16.5\end{array}$ & $\begin{array}{c}\mathrm{b} \\
0.02 \\
37.7 \\
\pm 21.1\end{array}$ & $\begin{array}{c}\mathrm{b} \\
7.37 \\
23.2 \\
\pm 11.4\end{array}$ & 0.29 & 22.21 & 0.335 & 15.56 \\
\hline T. test & 0.115 & 0.106 & $0.021 *$ & 0.152 & 0.685 & 0.13 & 0.079 & $0.027^{*}$ & 0.075 & $0.029 *$ & & & & \\
\hline
\end{tabular}

Mean with the same letter are not significantly different

$(*)$ significant at $\leq 0.05$

(**) highly significant at $\leq 0.01$

Table 13. Effects of Regimen diet with or without supplemented yogurt with cumin or lemon and Mixed Between them (1:1w/w)on Endothelium Function (Flow Dilation) in Female overweight and obese patients at baseline and after 12 weeks.

\begin{tabular}{|c|c|c|c|c|c|c|c|c|c|c|c|c|c|c|}
\hline \multirow{2}{*}{$\begin{array}{l}\text { Groups Treatment } \\
\text { Parameters } \mathrm{Mg} / \mathrm{dl}\end{array}$} & \multicolumn{2}{|c|}{$\begin{array}{c}\text { Group -1- } \\
\text { \# Control A } \\
\text { (usual diet) }\end{array}$} & \multicolumn{2}{|c|}{$\begin{array}{c}\text { Group -2- } \\
\text { \# Regimen diet }\end{array}$} & \multicolumn{2}{|c|}{$\begin{array}{c}\text { Group -3- } \\
\text { \# Regimen diet } \\
\text { containing cumin }\end{array}$} & \multicolumn{2}{|c|}{$\begin{array}{c}\text { Group -4- } \\
\text { \# Regimen diet } \\
\text { containing lemon }\end{array}$} & \multicolumn{2}{|c|}{$\begin{array}{c}\text { Group -5- } \\
\text { \# Regimen diet } \\
\text { containing cumin } \\
\text { and lemon }\end{array}$} & \multirow{2}{*}{$\begin{array}{c}\text { Anova } \\
\text { over- } \\
\text { weight }\end{array}$} & \multirow{2}{*}{$\begin{array}{c}\text { LSD } \\
\text { over- } \\
\text { weight }\end{array}$} & \multirow[t]{2}{*}{$\begin{array}{l}\text { Anova } \\
\text { obese }\end{array}$} & \multirow[t]{2}{*}{$\begin{array}{l}\text { LSD } \\
\text { obese }\end{array}$} \\
\hline & $\begin{array}{c}\text { Over- } \\
\text { weight }\end{array}$ & Obese & $\begin{array}{c}\text { Over- } \\
\text { weight }\end{array}$ & Obese & $\begin{array}{c}\text { Over- } \\
\text { weight }\end{array}$ & Obese & $\begin{array}{c}\text { Over- } \\
\text { weight }\end{array}$ & Obese & $\begin{array}{l}\text { Over- } \\
\text { weight }\end{array}$ & Obese & & & & \\
\hline $\begin{array}{l}\text { Endothelium Function } \\
\text { (Flow Dilation) at } \\
\text { baseline } \\
\text { (mean } \pm \mathrm{SD})\end{array}$ & $\begin{array}{r}15.9 \\
\pm 7.1\end{array}$ & $\begin{array}{c}26.3 \\
\pm 23.1\end{array}$ & $\begin{array}{c}12.6 \\
\pm 3.3\end{array}$ & $\begin{array}{c}17.4 \\
\pm 13.0\end{array}$ & $\begin{array}{c}13.8 \\
\pm 10.7\end{array}$ & $\begin{array}{c}10.1 \\
\pm 3.1\end{array}$ & $\begin{array}{r}13.9 \\
\pm 9.1\end{array}$ & $\begin{array}{c}9.1 \\
\pm 3.2\end{array}$ & $\begin{array}{c}9.2 \\
\pm 1.9\end{array}$ & $\begin{array}{l}13.6 \\
\pm 7.9\end{array}$ & 0.891 & - & 0.356 & - \\
\hline $\begin{array}{c}\text { Endothelium Function } \\
\text { (Flow Dilation) after } \\
12 \text { Weeks } \\
\text { (mean } \pm \mathrm{SD} \text { ) }\end{array}$ & $\begin{array}{c}\mathrm{a} \\
18.6 \\
6.2 \\
\pm 0.8\end{array}$ & $\begin{array}{c}a \\
23.03 \\
15.9 \\
\pm 12.0\end{array}$ & $\begin{array}{c}\mathrm{a} \\
14.8 \\
14.3 \\
+2.1 \\
\end{array}$ & $\begin{array}{c}a \\
19.66 \\
19.7 \\
\pm 11.6\end{array}$ & $\begin{array}{c}\mathrm{a} \\
14.29 \\
14.8 \\
\pm 8.9\end{array}$ & $\begin{array}{c}a \\
17.45 \\
12.2 \\
\pm 6.4\end{array}$ & $\begin{array}{c}\mathrm{ab} \\
13.15 \\
18.6 \\
\pm 7.8\end{array}$ & $\begin{array}{c}\mathrm{a} \\
15.9 \\
17.5 \\
\pm 8.7\end{array}$ & $\begin{array}{c}\mathrm{b} \\
3.73 \\
13.2 \\
\pm 11.4\end{array}$ & $\begin{array}{c}a \\
12.2 \\
23 \\
\pm 13.8\end{array}$ & 0.342 & 9.97 & 0.6 & 14.28 \\
\hline T. test & $0.011 *$ & 0.246 & 0.208 & 0.185 & 0.152 & $0.018^{*}$ & $0.000^{* *}$ & $0.001^{* *}$ & 0.067 & 0.106 & & & & \\
\hline
\end{tabular}

Mean with the same letter are not significantly different

$(*)$ significant at $\leq 0.05$

(**) highly significant at $\leq 0.01$ 
relationship documented between flavonoid consumption and heart disease risk factor as reducing blood pressure, improving flow-mediated dilation (FMD), improving weight management and dyslipidemia.

Oxidized LDL-s are a potent indicator of MCP-1 expression within the atherosclerotic plaque., HDL-s contain an enzyme, paraoxons, that is believed to confer protection against oxidation of LDL-c in the arterial wall [11] HDL Particals have multiple biological effects that could be attributed to their anti-atherothrombotic action including anti inflammatory, antioxidant, and profibrinolytic activities [13]. Low HDL-c levels increase the risk of coronary heart disease [7].

\section{Conclusion}

Supplemented yogurt with a mixed combination of lemon plus cumin $(1: 1 \mathrm{w} / \mathrm{w})$ raise the protective effect of either component alone in preventing atherosclerosis. Regular consumption of cumin mixed with lemon $(1: 1 \mathrm{w} / \mathrm{w})$ lead to reduction of total cholesterol, triglycerides, LDL, and leads to the elevation of brachial flow dilation improving also endothelial function and HDL-c.

\section{References}

[1]. Al-Shafy S.A.S, Seham A.M. Tharwat ,Ashraf. A. Abdel-Megeid, ;Randasalam and Rehab H. Gab Allah.(2020); The potential effects of lemon and cumin on weight reduction and blood sugar in obese and overweight patients. Egyptian Journal Of Nutrition; vol.35-No.2-(2020).

[2]. Dijk JM, Vander Graaf Y, Bots ML, Grobbee DE, Algra A. Carotid intimamedia thickness and the risk of new vascular events in patients with manifest atherosclerotic disease: the SMART study. Eur. Heart J. 2006; 27 (16): 1971-8. PMID: 16835264

[3]. Flagel KM, Kit BK, Orpana H, Graubard BI. Association of all cause mortality with overweight and obesity using standard body mass index categories: a systematic review and meta-analysis. JAMA. 2013; 309: 71-82. PMID: 23280227.

[4]. Flegal KM, Williamson DF, Pamuk ER, Rosenberg HM. Estimating deaths attributable to obesity in the united states. Am. J. Public Health. 2004;
94(9): 1486-9. PMID: 15333299.

[5]. Ma'had al-Taghdhiyah. Food Composition tables for Egypt. Nutrition Institute A.R.E, Egypt. 1996.

[6]. Miyake Y, Yamamoto K, Osawa T. Isolation of eriocitrin (eriodictyol 7-rutinoside) from lemon fruit (Citrus limon BURM. f.) and its antioxidative activity. Food Science and Technology International, Tokyo. 1997; 3(1): 84-9.

[7]. DJ Gordon, JL Probstfield, RJ Garrison, JD Neaton, WP Castelli, JD Knoke, et al. High-density lipoprotein-c and Cardiovascular disease. Circulation. 1989; 79 (1): 8-15. PMID: 2642759.

[8]. Hatch FT, Lees RS. Practical Methods for plasma lipoprotein analysis. Adv. Lipid Res. 1968; 6:1-68.

[9]. Hooper L, Kroon PA, Rimn EB, Gohn JS, Ian Harvey, Kathryn A Le Cornu, et al. Flavonoids, flavonoid-rich foods, and cardiovascular risk: a meta-analysis of randomized controlled trials. Am. J. Clin. Nutr. 2008; 88: 38-50. PMID: 18614722.

[10]. Lopes-Virella M, P Stone, S Ellis, J A Colwell. Cholesterol determination in high density lipoproteins separated by three different methods. Clin. Chem. 1977; 23: 882-884. PMID: 192488.

[11]. Mackness B, Hine D, Liu Y. Paraoxohase-1 inhibits oxidized LDL-induced MCP-1 production by endothelial cepps. Biochem Biophys Res. Commune. 2004; 318: 680-683.

[12]. Richmond W. Calorimetric method of determination of total cholest-erol and high density lipoprotein cholesterol. Clin. Chem. 1973; 19: 1350-1356.

[13]. Shah PK, Kaul S, Nilsson J, Cercek B. Exploiting the vascular protective effects of high-density lipoprotein and its apolipoproteins: an idea whose time for testing is coming, part I. Circulation. 2001; 104(19): 2376-2383. PMID: 11696481.

[14]. Twig G, Yaniv G, Levine H, Leiba A, Goldberger N, Derazne E, et al. Body mass index in 2.3 million adolescents and cardiovascular death in adulthood. N. Engl. J. Med. 2016; 374: 2430-2440.

[15]. Villar IC, Francis S, Webb A, Hobbs AJ. A Novel aspect of endothelium dependent regulation of vascular tone. Kidney Int. 2006; 70(5): 840-53. PMID: 16837917.

[16]. Wahalefeld AW. Colorimetric method of determination of triglycerides in serum or plasma. Academic Press, New York. 1974; 5: 183:1838.

[17]. Yaghmaie P, Parivar K, Haftsavar M. Effect of citrus aurantifolia peel essential oil on serum cholesterol levels in Wister rats. Jofpara medical science. 2011; 2(1): 29-32.

[18]. Yeboah J, Crouse JR, Hsu FC, Burke GL, Herrington DM. Brachial flowmediated dilation predicts incident cardiovascular health study. Circulation. 2007; 115(18): 2390-7. PMID: 17452608.

[19]. Zara R, Heshmati F, Fallahzadek H, Nadjarzadeh A. Effect of cumin powder on body composition and lipid profile in overweight and obese women. Complement there. Clin. Pract. 2014; 20(4): 297-301. 